\title{
Kimura Disease: Rare Manifestations in Unusual Populations
}

\author{
Aljoharah A Alshaikh1* ${ }^{*}$, Hebah M Musalem¹, Lin M Tuleimat ${ }^{1}$, and \\ Saad M Alajlan ${ }^{2}$ \\ ${ }^{1}$ Alfaisal University, P.0 Box 50927, Riyadh 11533, Saudi Arabia \\ ${ }^{2}$ Department of Dermatology, King Faisal Specialist Hospital and Research Center. P.O \\ Box 3354, Riyadh 11211, Kingdom of Saudi Arabia
}

\section{Case Report \\ Volume 2 Issue 2}

Received Date: April 05, 2017

Published Date: April 18, 2017

DOI: $10.23880 /$ cdoaj16000119

*Corresponding author: Aljoharah Al Shaikh, Alfaisal University, P.0 Box 50927, Riyadh 11533, Saudi Arabia, Tel:

0096650858 5860; E-mail: Aljoharah.alshaikh@gmail.com

\section{Abstract}

Kimura disease (KD) is a benign, rare, and chronic inflammatory disorder with an angiolymphatic proliferation of unknown etiology involving subcutaneous tissues, affecting a population sporadically. It is most common in middle-aged Asian men, as it is endemic in china and Japan. Herein, we present a medically free 40 years old Saudi female, with unusual clinical, radiological and histopathological manifestations of KD presented with extended facial swelling from the right to the left cheek with a bluish and firm mass in the center of the hard palate. Additionally, a solitary soft lymph node swelling was found in the anterior triangle of the neck. CT scan of the head and neck showed an area of nodularity and fat stranding in the left lower cheek. Histologically, hyperplastic changes of lymphatic tissues were viewed. Treatment with prednisolone showed transient improvement, thus Cyclosporine was suggested since it has a significant potential in decreasing the recurrences and the flare-ups of KD. Adequate reporting and documentation of those cases in the literature are strongly required to establish clear guidelines for the diagnosis and management of KD among Middle eastern population.

Keywords: Kimura Disease; Eosinophilia; Angiolymphatic proliferation; Saudi Arabia

\section{Abbreviation: KD: Kimura Disease}

\section{Introduction}

Kimura disease (KD) is a benign, rare form of chronic inflammatory disorder with angiolymphatic proliferation of unknown etiology involving subcutaneous tissues, affecting a population sporadically. It involves the subcutaneous tissue, predominantly in the head and neck and frequently associated with regional lymphadenopathy [1]. This disease has a predilection to Asian male descent and can stimulate neoplasm. Patients with KD usually present with the triad of a solitary enlarged painless lymph node or generalized lymphadenopathy (67\% to $100 \%$ ) eosinophilia and elevated serum levels of IgE [2]. KD has characteristic histological features that are important to recognize and can be used to differentiate it from hypersensitivity, drug reactions, and infections. These features include; follicular hyperplasia, eosinophilic infiltrations, and proliferation of post capillaries venules. KD can be confused with eosinophilic angiolymphatic hyperplasia [3]. Multiple modalities of treatment have been reported, such as surgical excision and adjuvant steroids, however, recurrence is well known to occur [4]. Herein, we present a case of KD who was diagnosed based on skin biopsies for a 42-year-old Saudi female who is currently following up with the dermatology department in King Faisal 
Specialist Hospital and Research Center (KFSHRC), Riyadh.

The literature is lacking sufficient documentation considering KD in the Middle Eastern population, which raises the importance to emphasize on reporting and documenting similar cases in the future, to increase our awareness of this disease, and to provide patients suffering from KD better care.

\section{Case Report}

This is a 40 years old Saudi female with no previous chronic medical illness or history of childhood complaint. 10 years ago (at the age of 30 ) she started complaining of progressively enlarged mid-frontal painless swelling. She underwent mid-frontal lesion excision 4 years ago in the national guard hospital in Jeddah. No more complaints regarding the frontal area. few months after her surgery she started complaining of progressively enlarging painless non-discharging swelling over the right side of the cheek and right lateral nasal side. One year back, she started complaining of extension of facial swelling to the left and right cheeks with a new hard palate mass. No discharge, new visual complaints, headache, functional nasal symptoms, airway symptoms or subjective fever.

Physical examination showed an old horizontal scar over the forehead, and right sided diffuse soft minimal dark swelling in both sides of the cheek involving the right and the left nasal dorsum. While examining the oral cavity, a $1 \times 1$ bluish and firm mass was found at the center of the hard palate (Figure1). Neck examination revealed a solitary painless, non-exudative, soft tissue swelling over the right side of the neck in the anterior triangle. Nasal endoscopy revealed bilateral grade 1 small nasal polyps on the left side of the sphenoethmoidal recess.

Investigations included; $\mathrm{CBC}$, PT, PTT, and chemistry, which were all within normal range. Beta HCG was done and it was negative as well. An ultrasound scan showed multiple heterogeneous hypoechoic lesions over the right cheek and the right lateral part of the neck. Non-enhanced and enhanced CT scans of the face were performed with sagittal and coronal views, which showed enhancing soft tissue masses and skin thickening involving both cheeks. They extend medially to the nasal bridge and the medial canthus bilaterally, however it is greater on the right side. There is an area of nodularity and fat stranding seen in the left lower cheek. There are scattered benign looking lymph nodes in the visualized part of the neck, as well as Intraparotid lymph nodal enlargement. There is minimal mucosal thickening of the right maxillary sinus and ethmoid air cells. An impression of Skin thickening with abnormal enhancing soft tissue involving the cheek, nose and medial periorbital region (Figure 2). Findings are suggestiveof an inflammatory process, and are less likely infectious CT of the neck with contrast report suggested multiple soft tissue venous malformation for clinical and histopathological correlation with KD. A true cut biopsy was sent to the histopathology which showed; skeletal muscle and fibroconnective tissue with increased eosinophils, which is a typical histological feature of KD. A true cut biopsy of the hard palate showed subepithelial fibrosis, surface keratosis, and reactive melanosis.

Differential diagnosis of KD, lymphoma, pseudotumor or Wegener granulomatosis were initially suspected. However, the collective clinical symptoms of painless enlarging subcutaneous masses of the right and left sided cheeks, Intra-parotid and cervical lymphadenopathy and peripheral eosinophilia, kept KD as the most likely diagnosis. Nodularity and fat stranding on facial CT, a central hard palate mass with subepithelial fibrosis, surface keratosis, and reactive melanosis were all unusually corresponding with KD. After initial therapy with Prednisolone $30 \mathrm{mg}$ in October 2016, transient improvement was noticed. Cyclosporine was suggested since it has a significant potential in decreasing the recurrences and the flare-ups of $\mathrm{KD}$. The patient is now following up with Dermatology department in KFSHRC monthly with an initial satisfactory outcome.

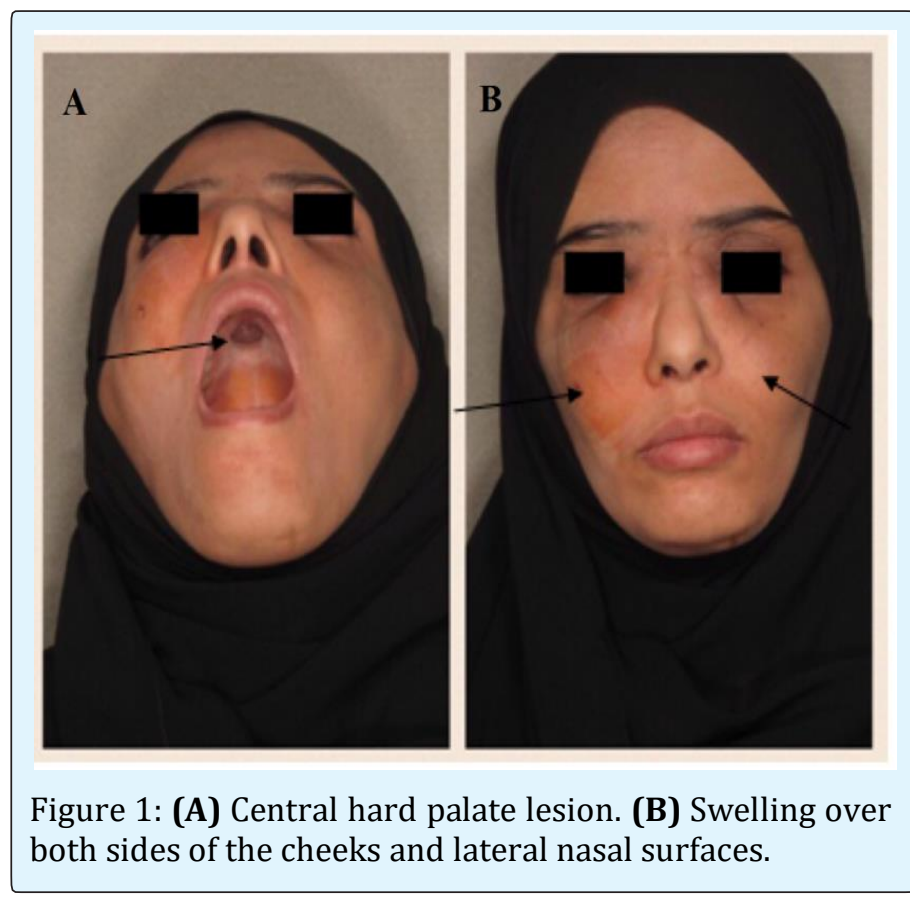




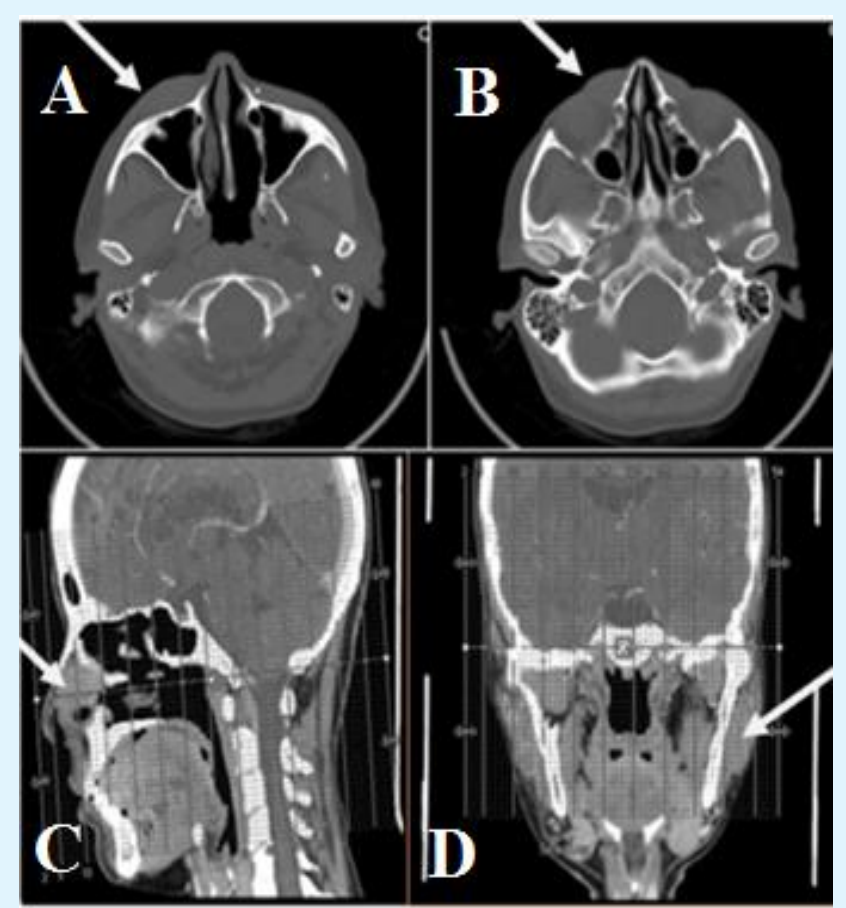

Figure 2: Nonenhanced and enhanced CT of the facial bone were performed with sagittal and coronal reformations in soft tissue and bone windows. A,B: Skin thickening with abnormal enhancing soft tissue involving the cheek, nose and medial periorbital region

C: Minimal mucosal thickening of the right maxillary sinus and ethmoid air cells D: Benign intraparotid lymph nodal enlargement.

\section{Discussion}

$\mathrm{KD}$ is a rare chronic inflammatory disorder with angiolymphatic proliferation, usually affecting Asian middle aged men. It was described in China in 1937 and the description of precise histological criteria was documented by Kimura et al in 1948 [5]. The theories developed to explain the pathology behind KD involves impairment or interference with immune regulation, atopic reaction to a persistent antigenic stimulus by arthropod bites, virus, and neoplasia [2]. Eosinophilia and increased serum IgE levels can help identify $\mathrm{KD}$ as a CD4(+) T-helper 2 (Th2) allergic reaction. Th2 cells would produce interleukins (IL)-4, IL-5, and IL-13; those interleukins act on $\mathrm{B}$ cells favoring the production of antigen-specific IgE. Therefore, Th2 cell over-expression of cytokines would play an indispensable role in the development of KD [6]. The diagnosis of KD is based on the following pathological criteria which includes; deep lymphoid infiltrate, reactive germinal centers, eosinophil infiltrate, stromal fibrosis, proliferation of postcapillary venules and vascularization of germinal centers $[7,8]$. Moreover, high PB eosinophil count can be used as a risk factor for recurrence in KD [9]. The most common involved sites include the parotid glands and the epitrochlear, axillary, and inguinal nodes. The masses usually enlarge gradually, otherwise, it is asymptomatic. Pruritus and dermatitis can ensue, and rare sites of involvement include the kidneys, orbits, ears, spermatic cord, nerves, and vessels.

As previously mentioned, KD has been typically seen among middle-aged Asian men peaked in the 3rd decade. However, in this case, the patient was a 40 -year-old middle eastern female. According to the literature, KD usually involves deep subcutaneous tissues and lymph nodes of the head and neck region with frequent regional lymphadenopathy or salivary gland enlargement, other sites of involvements includes the oral cavity, axilla, groin, and trunk. In this case, multiple lesions were found in typical sites, such as the nose, face, neck and sphenoethmoidal recess, yet others were rarely found in the hard palate. Additionally, enhanced CT imaging of the face showed unusual fat stranding over the subcutaneous tissue of the cheeks. Histologically, the biopsy of the hard palate showed atypical subepithelial fibrosis, surface keratosis, and reactive melanosis. Moreover, hyperplastic lymphatic changes in the intraparotid lymphnodal biopsy were viewed. The therapeutic approach for KD was not well documented in the literature, as most data are reported in case reports or short series. According to the literature, the treatment of KD is variable [5]. Surgical excisions have been designated to be the gold standard treatment for $\mathrm{KD}$, but recurrence is possible $[5,8]$. In patients with a single cutaneous lesion and no systemic signs, surgical excision was proposed as first line treatment with $100 \%$ of complete remission and $60 \%$ of relapse. However, for non-surgical KD or multiple lesions of $\mathrm{KD}$, immunosuppressive or immunomodulating therapy showing efficiency were oral corticosteroids, cyclosporine, interferon- $\alpha$ and thalidomide [4].

\section{Conclusion}

$\mathrm{KD}$ is one of the very rare medical diseases, especially in the middle east, in addition to the fact that the literature reported only a few cases of KD in the region of Saudi Arabia. The need of reporting more cases of KD with different clinical and histopathological presentations is crucial to establish clear guidelines for the diagnosis and management of KD among the Middle Eastern population. 


\section{References}

1. Kung IT, Gibson JB, Bannatyne PM (1984) Kimura's disease: a clinico-pathological study of 21 cases and its distinction from angiolymphoid hyperplasia with eosinophilia. Pathology 16(1): 39-44.

2. Fouda MA, Gheith O, Refaie A, El-Saeed M, Bakr A, et al. (2010) Kimura Disease: A Case Report and Review of the Literature with A New Management Protocol. Int J Nephrol 2010: 673908.

3. Kapoor NS, O'Neill JP, Katabi N, Wong RJ, Shah JP (2012) Kimura disease: diagnostic challenges and clinical management. Am J Otolaryngol 33(2): 259262.

4. Kottler D, Barète $S$, Quéreux G, Ingen-Housz-Oro S, Fraitag S, et al. (2015) Retrospective Multicentric Study of 25 Kimura Disease Patients: Emphasis on Therapeutics and Shared Features with Cutaneous IgG4-Related Disease. Dermatology 231(4): 367-377.
5. AlGhamdi FE, Al-Khatib TA, Marzouki HZ, AlGarni MA (2016) Kimura disease. No age or ethnicity limit. Saudi Med J 37(3): 315-319.

6. Banyameen I, Sawaimul K, Kambale (2015) T Kimura's disease embedding radial artery: A very rare presentation." Journal of Cancer Research and Therapeutics 11(4): 1031.

7. Chen H, Thompson LDR, Aguilera NSI, Abbondanzo SL (2004) Kimura disease: a clinicopathologic study of 21 cases. Am J Surg Pathol 28(4): 505-513.

8. Kim HT, Szeto C (1937) Eosinophilic hyperplastic lymphogranuloma, comparison with Mikulicz's disease. Chin Med J 23: 699-700.

9. Iwai H, Nakae K, Ikeda K, Ogura M, Miyamoto M, et al. (2007) Kimura disease: diagnosis and prognostic factors. Otolaryngol Head Neck Surg 137(2): 306-311. 